\title{
Desempeño de pruebas de no linealidad con la presencia de atípicos
}

\author{
Performance of non linearity test in the presence of outliers
}

Michael Vásqued

mvasquezl@unal.edu.co

\section{Resumen}

Evidencia sobre la existencia de estructuras no lineales en series de tiempo económicas se ha presentado en los últimos años en la literatura. Frecuentemente se usan pruebas para contrastar la hipótesis nula de linealidad; sin embargo la existencia de valores atípicos puede afectar su desempeño concluyendo de forma errónea que exista una no linealidad endógena en el proceso generador de datos. En este trabajo, mediante un ejercicio de simulación, se analiza el desempeño de tres pruebas para detectar no linealidad con y sin la presencia de atípicos. Asimismo, se examina si la serie de precios de la electricidad en Colombia tiene una dinámica no lineal.

Palabras clave: Series de tiempo; pruebas de linealidad, no linealidad, datos atípicos.

\begin{abstract}
Recently evidence about the existence of nonlinear structures in time series has been presented in the literature. Often tests are used to contrast linearity vs. nonlinearity. However the presence of outliers can seriously distort the results (concluding erroneously that is endogenous nonlinearity in the data generating process). Through a simulation study, it is analyze the performance of three tests of nonlinearity when there are outliers. In addition, it is analyze if the series of prices of electricity in Colombia presents a nonlinear endogenous dynamic.
\end{abstract}

Keywords: Time series, linearity tests, nonlinearity, outliers.

\section{Introducción}

En los últimos años en las ciencias económicas ha surgido el debate sobre la presencia de no linealidad en el proceso generador de datos de algunas series de tiempo.

${ }^{a}$ Universidad Nacional 
La no linealidad, desde un punto de vista empírico, surge por las múltiples interacciones estratégicas de los participantes del mercado que asumen actitudes de carácter no lineal hacia el riesgo y el retorno esperado de una inversión, ver Campbell et al. (1997).

En la literatura se encuentran múltiples pruebas para detectar la presencia de estructuras no lineales en series de tiempo univariadas. Las primeras pruebas se desarrollaron con base en el dominio de la frecuencia, ver Hinich (1982). Posteriormente se sugiere probar la no linealidad a partir de una descomposición de Volterra de la serie original, Keenan (1985) y Teräsvirta (1996). En los últimos años se han propuesto otras metodologías, una empleada comúnmente es el test BDS propuesto por Brock et al. (1996) que se basa en la integral de correlación. Esta última es una medida que cuantifica el número de veces que los patrones temporales se repiten en los datos. En Kyrtsou \& Serletis (2006) se exponen estas y otras metodologías empleadas en series de tiempo económicas.

Aunque el uso de pruebas estadísticas para determinar si la serie de tiempo evidencia efectivamente un comportamiento no lineal es extendido, la presencia de datos atípicos puede producir una no linealidad artificial o exógena que es independiente del proceso. Lo anterior puede distorsionar la mayoría de contrastes estadísticos rechazándose la hipótesis nula de linealidad.van Dijk et al. (1999b) muestran que la existencia de valores atípicos aditivos, particularmente en series cortas, afectan la correcta identificación de modelos autorregresivos de umbral. En este sentido proponen una prueba basada en estimación robusta para la correcta identificación de la no linealidad. En un artículo posterior, van Dijk et al. (1999a), concluyen que la existencia de este tipo de atípicos también conduce generalmente a rechazar la hipótesis nula de homocedasticidad, siendo verdadera, cuando se usa la prueba clásica de multiplicadores de Lagrange. De nuevo proponen el uso de una prueba robusta.

En este trabajo se explora mediante un ejercicio de simulación para un proceso autorregresivo de medias móviles (ARMA), cómo la presencia de distintos tipos de atípicos afectan el desempeño de las pruebas utilizadas para contrastar la hipótesis nula de linealidad. En particular se emplean el test propuesto por Keenan (1985), el de Teräsvirta (1996) y el test BDS.

Además se analiza la serie de los precios de la electricidad en el mercado colombiano. Esta serie ha sido ampliamente estudiada a través de distintas metodologías. Algunos autores han asumido que el proceso generador de datos es lineal. Otros, por el contrario, asumen la existencia de estructuras no lineales que pueden ser capturadas a través de modelos no paramétricos como redes neuronales. En este sentido, en este trabajo se pretende analizar si esta serie proviene de un proceso lineal, cuya dinámica se ve afectada por eventos exógenos, o existe un estructura no lineal endógena que justifique el uso de modelos no lineales para obtener un 
mejor pronóstico. A continuación se exponen algunos trabajos que previamente han modelado esta serie.

Lira et al. (2009) proyecta el precio del día siguiente teniendo en cuanta varios escenarios de demanda (alto, medio y bajo), construye una red neuronal multivariada usando como variables de entrada el precio, niveles diarios de embalse, precios de los combustibles y precio del gas. Velásquez (2008) propone una amplia gama de modelos para pronosticar el precio de la energía eléctrica. Usa un modelo autorregresivo con variables explicativas, redes neuronales, regresión de transición suave (STR), un modelo no paramétrico de regresión no lineal (ANFIS) y modelos de componentes no observables. Mediante un análisis de residuales se observa la superioridad de las especificaciones no lineales.

Velásquez \& Franco (2010) compara un modelo VEC con una red neuronal dinámica, encontrando una mejor precisión en esta última especificación. Barrientos et al. (2012) compara un modelo de vector de corrección de errores (VEC) y uno basado en redes neuronales. Los resultados sugieren la superioridad del modelo VEC. Tabarez (2017) compara el pronóstico por fuera de la muestra de un modelo autorregresivo de media móvil y una red neuronal. Usando el test de Diebold-Mariano se concluye que no existe diferencia significativa en la exactitud de la predicción de los dos modelos. Finalmente, cabe resaltar el trabajo de Castaño \& Sierra (2012). A partir de la serie mensual del precio de la electricidad se analiza si existe una tendencia determinística, una raíz unitaria o la presencia de distintos cambios de nivel, ocasionados por factores climáticos o regulatorios. Los autores encuentran evidencia de un proceso estacionario alrededor de varios cambios de nivel.

\section{Modelación de series de tiempo}

\subsection{Linealidad y no linealidad de series de tiempo}

Según Brock \& de Lima (1995) dada una serie de tiempo, $\left\{x_{t}\right\}$, que es función de una sucesión de innovaciones $\varepsilon_{t}$, independientes e idénticamente distribuidas ( iid), se dice que la serie es lineal si puede expresarse, mediante la descomposición de Wold, como una combinación lineal de coeficientes constantes de las innovaciones

$$
x_{t}=\sum_{i=1}^{\infty} a_{i} \varepsilon_{t-i} .
$$

Siguiendo esta misma línea, Campbell et al. (1997) asumen que existe una función $f$ que relaciona las $x_{t}$ con sus innovaciones

$$
x_{t}=f\left(\varepsilon_{t}, \varepsilon_{t-1}, \varepsilon_{t-2}, \ldots, \varepsilon_{t-j}\right) .
$$


La serie se puede descomponer mediante una expansión de Taylor alrededor de $\varepsilon_{t}=0$, para $\varepsilon_{t-1}, \varepsilon_{t-2}, \ldots$ dados, como

$$
x_{t}=f\left(0, \varepsilon_{t-1}, \ldots\right)+\varepsilon_{t} \frac{d}{d t} f\left(0, \varepsilon_{t-1}, \ldots\right)+\frac{1}{2} \varepsilon_{t}^{2} \frac{d^{2}}{d t} f\left(0, \varepsilon_{t-1}, \ldots\right)+\ldots
$$

Si la magnitud de la innovación $\varepsilon_{t}$ es pequeña, los términos de orden superior resultan despreciables, y (3) se puede aproximar por

$$
x_{t}=g\left(0, \varepsilon_{t-1}, \ldots\right)+\varepsilon_{t} h\left(0, \varepsilon_{t-1}, \ldots\right),
$$

donde $g\left(0, \varepsilon_{t-1}, \ldots\right)=f\left(0, \varepsilon_{t-1}, \ldots\right)$ y $h\left(0, \varepsilon_{t-1}, \ldots\right)=\frac{d}{d t} f\left(0, \varepsilon_{t-1}, \ldots\right)$. Dado que $E\left[x_{t} \mid x_{t-1}, x_{t-2, . .}\right]=g\left(\varepsilon_{t-1}, \varepsilon_{t-2}, \ldots\right)$, la función $g$ representa la media condicional del proceso y $h^{2}$ la varianza condicional Teräsvirta et al. (1994). Los modelos que usan la función $g$ no lineal se denominan modelos no lineales en media, mientras que los modelos que usan una función $h^{2}$ no lineal, son llamados modelos no lineales en varianza Tsay (2001). Según Hsieh (1995) la forma en la que se relacione la innovación con la función $f$ no lineal, determinará el tipo de no linealidad.

En series no lineales la media condicional se puede descomponer en una componente lineal y una no lineal, expandiendo la función $g$ en una serie de Volterra en la forma

$g\left(\varepsilon_{t-1}, \varepsilon_{t-2} \ldots\right)=\sum_{i=1}^{\infty} a_{i} \varepsilon_{t-i}+\sum_{i=1}^{\infty} \sum_{j=i}^{\infty} b_{i j} \varepsilon_{t-i} \varepsilon_{t-j}+\sum_{i=1}^{\infty} \sum_{j=i}^{\infty} \sum_{k=j}^{\infty} c_{i j k} \varepsilon_{t-i} \varepsilon_{t-j} \varepsilon_{t-k}+\ldots$

El primer término es una media móvil lineal, mientras que los demás son no lineales. Un test para probar dependencia no lineal en la media condicional es el test propuesto por Keenan (1985) que se basa en la expansión de Volterra de segundo orden

$$
x_{t}=\mu+\sum_{i=-\infty}^{\infty} \theta_{i} a_{t-i}+\sum_{i, j=-\infty}^{\infty} \theta_{i, j} a_{t-i} a_{t-j} .
$$

$x_{t}$ es no lineal si cualquier coeficiente de orden alto es distinto de cero. Keenan (1985) propone contrastar la hipótesis nula de linealidad para un proceso estacionario de media cero, de modo que se ajusta un modelo autorregresivo a los datos originales. Si $\widehat{x}_{t}$ son los valores ajustados, $\widehat{a}_{t}$ los residuales y $S S E_{e}=\sum a^{2}(t)$ la suma de los residuales al cuadrado, se ajusta la regresión

$$
\widehat{x}_{t}=\alpha_{0}+\sum_{i} \alpha_{i} x_{t-i}+v_{t} \quad \text { para } i=1 \ldots m
$$


se calculan los residuales $v(t)$ y la suma de residuales al cuadrado $S S E_{v}$. Finalmente, se regresa $e_{t}=\beta v(t)+w(t)$ y se calcula el estadístico

$$
T_{n}=\frac{\beta^{2} S S E_{v}(T-2 m-2)}{\left(S S E_{e}\right)-\left(\beta^{2} S S E_{v}\right)} .
$$

Bajo hipótesis nula de linealidad $T_{n}$ distribuye asintóticamente como $F(1, n-$ $2 M-2)$.

La prueba anterior asume un proceso generador de datos lineal. Teräsvirta (1996) considera el siguiente modelo

$$
x_{t}=\phi\left(\gamma^{\prime} \omega_{t}\right)+\pi^{\prime} \omega_{t}+u_{t}
$$

donde $u_{t} \sim i i d\left(0, \sigma^{2}\right), \omega_{t}=\left(1, \widetilde{\omega}_{t}^{\prime}\right)^{\prime}, \widetilde{\omega}_{t}=\left(x_{t-1}, \ldots, x_{t-p}\right)^{\prime}, \pi=\left(\pi_{0}, \pi_{1}, \ldots, \pi_{p}\right)$ y $\gamma=\left(\gamma_{0}, \gamma_{1}, \gamma_{2}, \ldots, \gamma_{p}\right)$.

Si se define $\omega_{t}=\theta_{0} \varphi\left(\gamma^{\prime} \omega_{t}\right)$, donde $\varphi\left(\gamma^{\prime} \omega_{t}\right)=\left\{1+\exp \left(-\gamma^{\prime} \omega_{t}\right)\right\}^{-1}-1 / 2$, la especificación (9) es un modelo autorregresivo no lineal del tipo red neuronal. Bajo la hipótesis nula de que $x_{t}$ es lineal, es decir $x_{t}=\pi^{\prime} \omega_{t}+u_{t}$, la hipótesis puede definirse como $H_{0}: \gamma=0$ contra la alternativa $\gamma \neq 0$.

Dado que (9) no es identificable bajo hipótesis nula, se realiza una expansión de Volterra de tercer orden alrededor de $\gamma=0$. Teräsvirta (1996) muestra que (9) se redefine como

$$
x_{t}=\widetilde{\pi}^{\prime} \omega_{t}+\sum_{i=1}^{p} \sum_{j=i}^{p} \delta_{i j} x_{t-i} x_{t-j}+\sum_{i=1}^{p} \sum_{j=i}^{p} \sum_{k=j}^{p} \delta_{i j k} x_{t-i} x_{t-j} x_{t-k}+\widetilde{u}_{t}
$$

donde $\delta_{i j}=d_{i j} \theta_{0} \gamma_{i} \gamma_{j} \gamma_{0}$ con $d_{i j}=1 / 36$ si $i=j$ y $d_{i j}=1 / 18$ en otro caso. $\delta_{i j k}=d_{i j k} \theta_{0} \gamma_{i} \gamma_{j} \gamma_{k}$ con $d_{i j k}=1 / 36$ si $i=j=k, d_{i j k}=1 / 18$ si $i=j, i=k$ o $k=j$ y $d_{i j k}=1 / 6$ en otro caso. La hipótesis nula se define como

$$
H_{0}^{\prime}: \delta_{i j}=0, \delta_{i j k}=0, \quad \text { para } i=1, \ldots, p ; j=1, \ldots, p ; k=1, \ldots, p
$$

La prueba se lleva a cabo en tres etapas. Se regresa $x_{t}$ sobre $1, x_{t-1}, x_{t-2}, \ldots, x_{t-p}$ y se computa la suma de residuales al cuadrado $S S R_{0}=\sum \widehat{u}_{t}^{2}$. A continuación se regresa $\widehat{u}_{t}$ sobre $1, x_{t-1}, x_{t-2}, \ldots, x_{t-p}$ y $m$ regresores auxiliares obteniendo $S S R=\sum \widehat{v}_{t}^{2}$. Finalmente se computa el estadístico

$$
F=\frac{\left(S S R_{0}-S S R\right) / m}{S S R /(T-p-1-m)}
$$

Bajo la hipótesis de linealidad $F$ distribuye asintóticamente $F(m, T-p-1-m)$. 
Otra prueba empleada comúnmente para determinar se existe una estructura no lineal en la media condicional es el test propuesto por Brock et al. (1996) denominado test BDS. El test fue propuesto originalmente para determinar si las observaciones de una serie de tiempo son independientes e idénticamente distribuidas $(i i d)$ detectando todo tipo de dependencia lineal o no lineal. Si $\left\{x_{t}\right\}$ es una serie de tiempo compuesta por observaciones independientes e idénticamente distribuidas ( iid), la probabilidad de que una historia de dos observaciones estén a una distancia menor o igual a $\varepsilon$ la una de la otra, está dada por

$$
\begin{gathered}
C_{2}(\varepsilon)=P\left(\left|x_{t}-x_{j}\right| \leq \varepsilon,\left|x_{t-1}-x_{j-1}\right| \leq \varepsilon\right) \\
=P\left(\left|x_{t}-x_{j}\right| \leq \varepsilon\right) P\left(\left|x_{t-1}-x_{j-1}\right| \leq \varepsilon\right) \\
=P\left(\left|x_{t}-x_{j}\right| \leq \varepsilon\right)^{2} \\
=\left[C_{1}(\epsilon)\right]^{2} .
\end{gathered}
$$

Generalizando 16 a un espacio de fase $m$-dimensional, se tiene

$$
C_{m}(\epsilon)=\left[C_{1}(\epsilon)\right]^{m}
$$

donde $C_{m}(\epsilon)$ es la integral de correlación de Gassberger \& Procaccia (1983) cuyo estimador, para una serie de longitud $n$, es

$$
C_{m n}(\epsilon)=\frac{2}{(n-m+1)(n-m)} \sum_{s=1}^{n-m+1} \sum_{t=1}^{n-m+1} \prod_{s=1}^{m-1} H\left(\varepsilon-\left|x_{s+j}-x_{t+j}\right|\right)
$$

donde $H(R)=\left\{\begin{array}{cc}1 & \text { si } \varepsilon-\left|x_{s+j}-x_{t+j}\right| \geq 0 \\ 0 & \text { eoc }\end{array}\right.$, es la función heaviside que enumera el número de $m$-dimensiones que cumplen con la condición de cercanía. De este modo el estadístico BDS se define como

$$
W_{m}(\epsilon)=\sqrt{T} \frac{C_{m n}(\epsilon)-\left[C_{1}(\epsilon)\right]^{m}}{\widehat{\sigma}_{m}(\epsilon)}
$$

donde $\widehat{\sigma}_{m}(\epsilon)$ es el estimador asintótico de la desviación estándar de $C_{m n}(\epsilon)-$ $\left[C_{1}(\epsilon)\right]^{m}$. Bajo la hipótesis nula de datos $i i d$, el test BDS converge a una distribución normal estándar. Una descripción detallada del test y sus propiedades se puede encontrar en Brock et al. (1996). Para probar dependencia no lineal el BDS se calcula sobre los residuales de un modelo que remueva la dependencia lineal de los datos.

Comunicaciones en Estadística, junio 2020, Vol. 13, No. 1 


\subsection{Modelo ARIMA}

Un modelo empleado tradicionalmente en el pronóstico de series de tiempo ha sido el propuesto por Box \& Jenkins (1970) conocido como Modelo Autorregresivo de Medias Móviles Integrado (ARIMA). Para una serie de tiempo $\left\{x_{t}, t=1, \ldots, T\right\}$, el modelo $A R I M A(p, d, q)$ se define como

$$
\phi(L)(1-L)^{d} x_{t}=\theta(L) \epsilon_{t},
$$

donde $L$ denota el operador de retardo, $d$ el parámetro de diferenciación, $\phi(L)=$ $1-\phi_{1} L-\ldots-\phi_{P} L^{p}$ y $\theta(L)=1-\theta_{1} L-\ldots-\theta_{q} L^{q}$ son los polinomios autorregresivos y de medias móviles, respectivamente. Si $\left\{x_{t}\right\}$ es estacionario e invertible, las raíces de ambos polinomios igualados a cero se encuentran fuera del círculo unidad, ademas los errores aleatorios $\epsilon_{t}$ son independientes e idénticamente distribuidos normales, con media cero y varianza $\sigma^{2}$.

El parámetro de diferenciación es un número entero positivo, generalmente $|d| \leq 2$. Este parámetro surge como consecuencia de la existencia de series que no satisfacen la condición de estacionariedad en media. Sin embargo, algunas series pueden volverse estacionarias al ser diferenciadas una o más veces. Es decir, si se diferencia una vez la serie no estacionaria $x_{t}$, se puede obtener una nueva serie que cumpla esta propiedad $\nabla x_{t}=x_{t}-x_{t-1}$.

Si $d=1$, el proceso puede ser persistente. La persistencia es entendida como el efecto permanente de un cambio unitario en la innovación sobre el nivel futuro (pronóstico) de la serie 1 . En este caso, se dice que el proceso tiene memoria infinita. Si $d=0$, el modelo se reduce a un $\operatorname{ARMA}(p, q)$ con memoria corta. Para determinar los órdenes de los polinomios autorregresivos y de medias móviles se sigue el procedimiento de modelación proporcionado por Box \& Jenkins (1970).

\subsection{Modelación con datos atípicos}

La efectividad del modelo ARIMA requiere que el proceso presente cierta homogeneidad o estabilidad a través del tiempo. Frecuentemente el proceso es sometido a cambios de carácter transitorio o permanente de tipo exógeno. Cuando se conoce el evento histórico que cambia la dinámica del proceso se incorpora como una variable regresora de intervención; sin embargo, cuando este se desconoce, se define como un dato atípico. Para una correcta identificación, estimación y pronóstico del modelo es necesario incorporar los atípicos en la especificación del modelo. En

\footnotetext{
${ }^{1}$ Como lo señala Cochrane (1991), la existencia de una raíz unitaria no es sinónimo de persistencia. En este sentido, de manera informal sería más adecuado decir que una serie que tenga una probabilidad mayor de rechazar la prueba de raíz unitaria generalmente puede tener menos persistencia.
}

Comunicaciones en Estadística, junio 2020, Vol. 13, No. 1 
este sentido, Tsay (1986) y Chen \& Liu (1993) proponen una metodología para el tratamiento de datos atípicos. Para incorporar el efecto de múltiples datos atípicos se redefine 20 como

$$
x_{t}^{\prime}=x_{t}+\sum_{i=1}^{k} w_{i} V_{i}(L) I_{t}\left(t_{i}\right)
$$

donde $x_{t}$ es la serie sin atípicos y sigue un proceso $A R I M A, I_{t}(t)$ es una función indicadora toma el valor $I_{t}(t)=1$ si $t=t_{i}$ y $I_{t}(t)=0$ en otro caso, $w$ es la magnitud del atípico. Dependiendo del tipo de atípico el polinomio $V(L)$ adopta varias especificaciones. En la literatura se distinguen cuatro tipos de atípicos:

- Atípico aditivo (AO): afecta solo una observación, en este caso $V(L)=1$.

- Atípico innovacional (IO): afecta solo una innovación, en este caso $V(L)=$ $\theta(L) /(1-L)^{d} \phi(L)$

- Cambio temporal (TC): es un cambio que afecta todas las observaciones después del instante en que ocurre pero decrece en forma exponencial. En este caso $V(L)=1 /(1-\delta L)$, generalmente $\delta=0.7$

- Cambio de tendencia (LS): es un cambio en la tendencia lineal de las serie afectando todas las observaciones. En este caso $V(L)=1 / 1-L$.

En la gráfica 1 se muestra el efecto de cada atípico sobre un modelo $A R(1)$ simulado. En la ecuación 21) $x_{t}$ sigue un proceso ARIMA que despejando de 20 podemos expresar como

$$
x_{t}=\frac{\theta(L) \epsilon_{t}}{\phi(L)(1-L)^{d}} .
$$

Si se multiplican ambos lados de la ecuación 21 por $\Pi(L)=\frac{\phi(L)(1-L)^{d}}{\theta(L)}$ se obtine un modelo de regresión común de la forma

$$
\widehat{e}_{t}=\sum_{i=1}^{k} w_{i} \Pi(L) V_{i}(L) I_{t}\left(t_{i}\right)+\epsilon_{t},
$$

donde $\widehat{e}_{t}=\Pi(L) x_{t}^{\prime}$. Los parámetros $w_{i}$ pueden ser estimados por MCO dado que $\epsilon_{t}$ es iid. Asimismo se puede contrastar la hipótesis nula de que cada parámetro es igual a cero con el estadístico usual $\widehat{T}_{i}=\widehat{w}_{i} / \operatorname{se}\left(\widehat{w}_{i}\right)$.

Dado que no se conoce el instante en el que ocurre el dato atípico, para cada observación $t=1, \ldots, T$ se deben calcular los coeficientes $\widehat{w}_{I O}(t), \widehat{w}_{A O}(t), \widehat{w}_{T C}(t)$, $\widehat{w}_{L S}(t)$ y sus respectivos estadísticos $\widehat{T}_{I O}(t), \widehat{T}_{A O}(t), \widehat{T}_{T C}(t)$ y $\widehat{T}_{L S}(t)$. Para determinar el tipo de dato atípico se escoge el máximo $\lambda_{T}=\max \left\{\left|\widehat{T}_{I O}(t)\right|,\left|\widehat{T}_{A O}(t)\right|\right.$, $\left|\widehat{T}_{T C}(t)\right|,\left|\widehat{T}_{L S}(t)\right|$. 

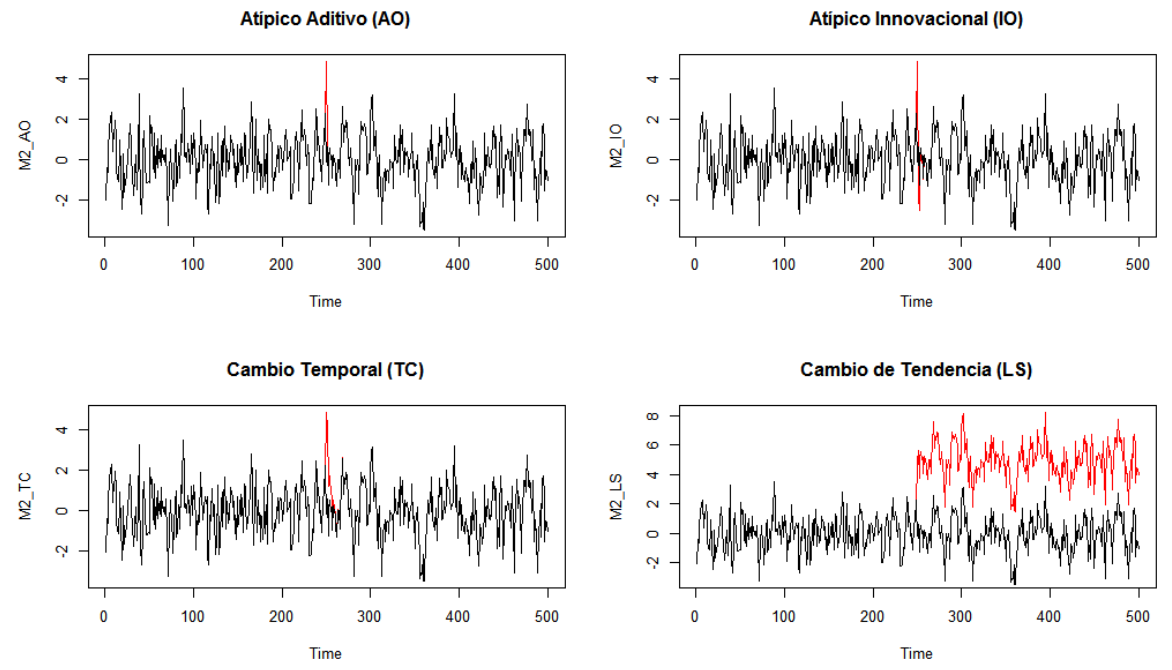

Figura 1: Efecto de un dato atípico sobre el modelo $A R(\phi=0.5)$. La línea negra corresponde a la serie original y la línea roja incorporando el dato atipico.

En la práctica tampoco se conocen los parámetros del modelo. Chen \& Liu (1993) 2 proponen el siguiente procedimiento iterativo. En una primera etapa se identifica y se estima un modelo $A R I M A$ por máxima verosimilitud exacta para la serie observada. Se estiman los estadísticos $\widehat{T}_{i}$, usando los residuales de este primer modelo. Si se encuentran datos atípicos significativos se elimina su efecto de las observaciones y de los residuales según sea su tipo. En una segunda etapa con los posibles puntos identificados como datos atípicos, se estima conjuntamente su tamaño $\widehat{w}_{i}$ usando la regresión (23). De nuevo se computan los estadísticos $\widehat{T}_{i}$ y se eliminan aquellos que no estén dentro de la región critica. Se obtiene una serie ajustada eliminando el efecto de los atípicos obtenidos en el paso anterior. Se estima de nuevo el modelo por máxima verosimilitud, si la variación de la suma de los residuales al cuadrado respecto a la estimación anterior es mayor a un error fijado, se inicia de nuevo la etapa dos. En la etapa final, se obtienen los residuales del modelo anterior y se repite la etapa uno y dos para obtener la magnitud de los atípicos encontrados y con estos corregir la serie original.

\footnotetext{
${ }^{2}$ Para llevar a cabo el procedimiento se utiliza la librería tsoutlers. Detalles computacionales se pueden encontrar en López de Lacalle (2017).
}

Comunicaciones en Estadística, junio 2020, Vol. 13, No. 1 
Tabla 1: Simulación sin atípicos

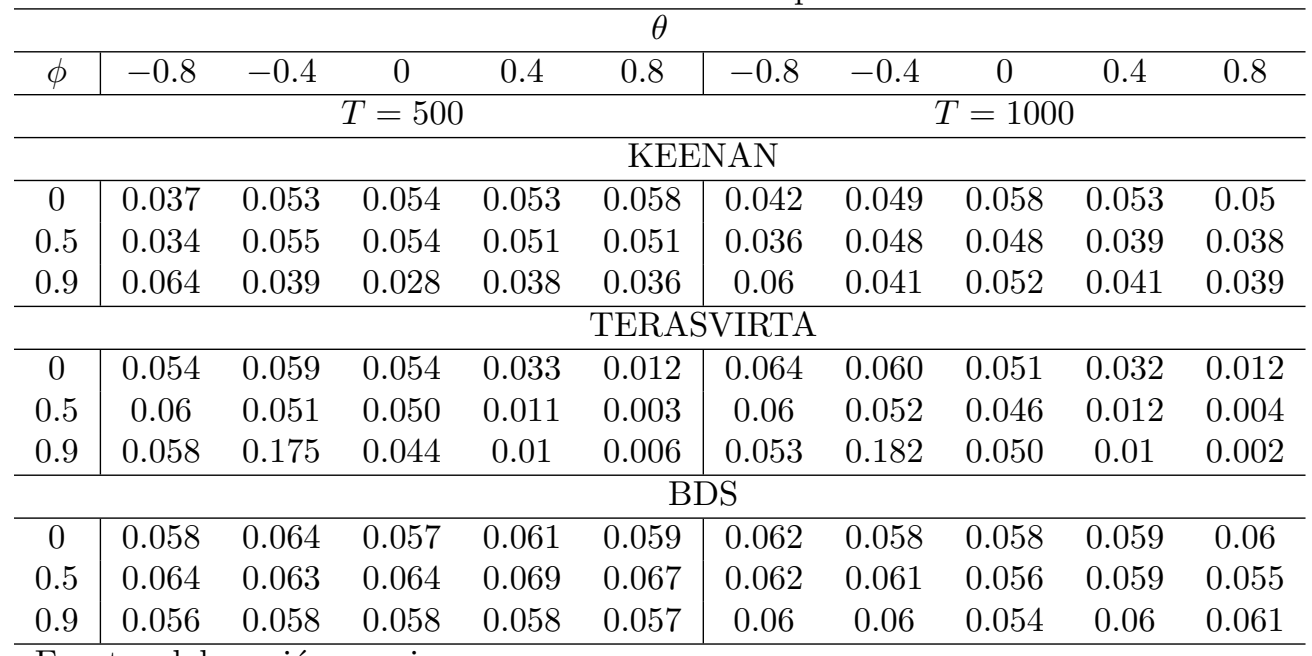

Fuente: elaboración propia.

\section{Experimento Monte Carlo}

Para analizar el desempeño se los contrastes de Keenan (1985), Teräsvirta (1996) y test BDS cuando existen datos atípicos, se simula el modelo $A R M A(1,1)$

$$
(1-\phi L) Z_{t}=(1-\theta L) a_{t}
$$

para los valores $\phi=0 ; 0.5 ; 0.9$ y $\theta=-0.8 ;-0.4 ; 0 ; 0.4 ; 0.8$. $a_{t}$ sigue una distribución $N(0,1)$. Se realizan simulaciones para los tamaños $T=500$ y $T=1000$. El número de repeticiones fue de 2500 y el nivel de significancia para las pruebas fue de $\alpha=0.053$

En la Tabla 1 se muestran los resultados obtenidos para la simulación sin datos atípicos. Para cada contraste se muestra la razón entre el número de veces que se rechaza la hipótesis nula de linealidad sobre el total de pruebas realizadas. Valores cercanos a cero indican que el contraste aceptó para la mayoría de los modelos simulados la hipótesis nula de linealidad. Por el contrario, un valor cercano a uno indica que en promedio la prueba rechaza la hipótesis nula de linealidad en la mayoría de los casos. Como puede verse, tanto para $T=500$ como para $T=1000$, los contrastes de Keenan y Terasvirta se acercan en general al valor nominal de 0.05. Se observa que la prueba de Keenan tiene en promedio un mejor desempeño cuando existen coeficientes grandes y negativos en la componente de medias móviles. El test BDS tiene un comportamiento bastante estable, los valores oscilan en el rango de 0.05 y 0.06 .

En la Tabla 2 se presenta la simulación con un dato atípico aditivo (AO). Clara-

\footnotetext{
${ }^{3} \mathrm{El}$ código computacional se puede encontrar en el anexo.
} 
Tabla 2: Simulación con atípico aditivo (AO)

\begin{tabular}{c|ccccc|ccccc}
\hline \multicolumn{10}{c}{$\theta$} \\
\hline$\phi$ & -0.8 & -0.4 & 0 & 0.4 & 0.8 & -0.8 & -0.4 & 0 & 0.4 & 0.8 \\
\hline \multicolumn{10}{c}{$T=500$} \\
\hline \multicolumn{10}{c}{ KEENAN } \\
\hline 0 & 0.737 & 0.200 & 0.039 & 0.122 & 0.192 & 0.704 & 0.152 & 0.047 & 0.096 & 0.149 \\
0.5 & 0.154 & 0.051 & 0.166 & 0.347 & 0.379 & 0.133 & 0.047 & 0.120 & 0.256 & 0.310 \\
0.9 & 0.06 & 0.056 & 0.071 & 0.127 & 0.172 & 0.054 & 0.052 & 0.077 & 0.098 & 0.154 \\
\hline \multicolumn{10}{c}{ TERASVIRTA } \\
\hline 0 & 0.242 & 0.219 & 0.051 & 0.200 & 0.216 & 0.217 & 0.191 & 0.053 & 0.172 & 0.164 \\
0.5 & 0.1 & 0.064 & 0.399 & 0.398 & 0.198 & 0.108 & 0.066 & 0.318 & 0.280 & 0.123 \\
0.9 & 0.072 & 0.542 & 0.296 & 0.127 & 0.046 & 0.071 & 0.456 & 0.213 & 0.070 & 0.022 \\
\hline \multicolumn{10}{c}{ BDS } \\
\hline 0 & 0.308 & 0.075 & 0.060 & 0.077 & 0.332 & 0.235 & 0.069 & 0.055 & 0.061 & 0.231 \\
0.5 & 0.079 & 0.064 & 0.074 & 0.156 & 0.647 & 0.06 & 0.057 & 0.063 & 0.084 & 0.483 \\
0.9 & 0.059 & 0.086 & 0.093 & 0.232 & 0.832 & 0.063 & 0.067 & 0.0745 & 0.135 & 0.670 \\
\hline
\end{tabular}

Fuente: elaboración propia.

mente los contrastes de Keenan y Terasvirta se alejan de su valor nominal y se incrementa la probabilidad de error tipo uno. No se observan diferencias significativas cuando se incrementa el tamaño. El contraste de Keenan registra el peor desempeño para el modelo $A R M A(\phi=0, \theta=-0.8)$ mientras que el de Terasvirta en $\operatorname{ARMA}(\phi=0.9, \theta=-0.4)$. El test BDS registra el mejor desempeño acercándose incluso al valor nominal de 0.05 en algunos casos.

En la Tabla 3 se muestran los resultados de las pruebas cuando se incluye un dato atípico innovacional (IO). En general se observa que los contrastes de Keenan y Terasvirta son inestables y específicamente para el modelo $A R M A(\phi=0, \theta=$ $-0.8)$ ambas pruebas registran tamaño promedio cercano a la unidad. De nuevo el test BDS tiene en promedio el mejor despeño; sin embargo cuando los coeficientes de medias móviles son altos la prueba registra un deterioro significativo.

Los resultados de la simulación incorporando un cambio temporal (TC) se presentan en la Tabla 4. Se observa que el error en las pruebas se incrementa de forma generalizada para todos los órdenes y, de nuevo, es especialmente alto cuando los coeficientes de medias móviles son grandes y negativos.

Finamente en la tabla Tabla 5 se observan los resultados de los contrastes cuando se incorpora un cambio de tendencia (TS). Claramente el test de Terasvirta es el que tiene el peor desempeño alcanzando un tamaño promedio de uno, evidenciando que en todos los casos se rechaza erróneamente la hipótesis nula de linealidad. 
Tabla 3: Simulación con atípico innovacional (IO)

\begin{tabular}{|c|c|c|c|c|c|c|c|c|c|c|}
\hline \multicolumn{11}{|c|}{$\theta$} \\
\hline$\phi$ & -0.8 & -0.4 & 0 & 0.4 & 0.8 & -0.8 & -0.4 & 0 & 0.4 & 0.8 \\
\hline & \multicolumn{5}{|c|}{$T=500$} & \multicolumn{5}{|c|}{$T=1000$} \\
\hline & \multicolumn{10}{|c|}{ KEENAN } \\
\hline 0 & 0.999 & 0.568 & 0.039 & 0.265 & 0.132 & 1.000 & 0.462 & 0.046 & 0.193 & 0.111 \\
\hline 0.5 & 0.587 & 0.066 & 0.470 & 0.438 & 0.382 & 0.609 & 0.051 & 0.333 & 0.333 & 0.550 \\
\hline 0.9 & 0.072 & 0.119 & 0.231 & 0.254 & 0.289 & 0.064 & 0.085 & 0.172 & 0.280 & 0.395 \\
\hline \multicolumn{11}{|c|}{ TERASVIRTA } \\
\hline 0 & 0.828 & 0.695 & 0.051 & 0.694 & 0.822 & 0.777 & 0.644 & 0.053 & 0.062 & 0.747 \\
\hline 0.5 & 0.397 & 0.102 & 0.842 & 0.761 & 0.596 & 0.388 & 0.106 & 0.731 & 0.669 & 0.508 \\
\hline 0.9 & 0.116 & 0.720 & 0.446 & 0.280 & 0.183 & 0.126 & 0.634 & 0.357 & 0.200 & 0.120 \\
\hline \multicolumn{11}{|c|}{ BDS } \\
\hline 0 & 0.770 & 0.136 & 0.059 & 0.137 & 0.790 & 0.623 & 0.09 & 0.055 & 0.0857 & 0.628 \\
\hline 0.5 & 0.184 & 0.066 & 0.095 & 0.363 & 0.980 & 0.125 & 0.055 & 0.074 & 0.187 & 0.943 \\
\hline 0.9 & 0.061 & 0.175 & 0.105 & 0.659 & 1.000 & 0.062 & 0.109 & 0.079 & 0.369 & 0.980 \\
\hline
\end{tabular}

Fuente: elaboración propia.

Tabla 4: Simulación con cambio temporal (TC)

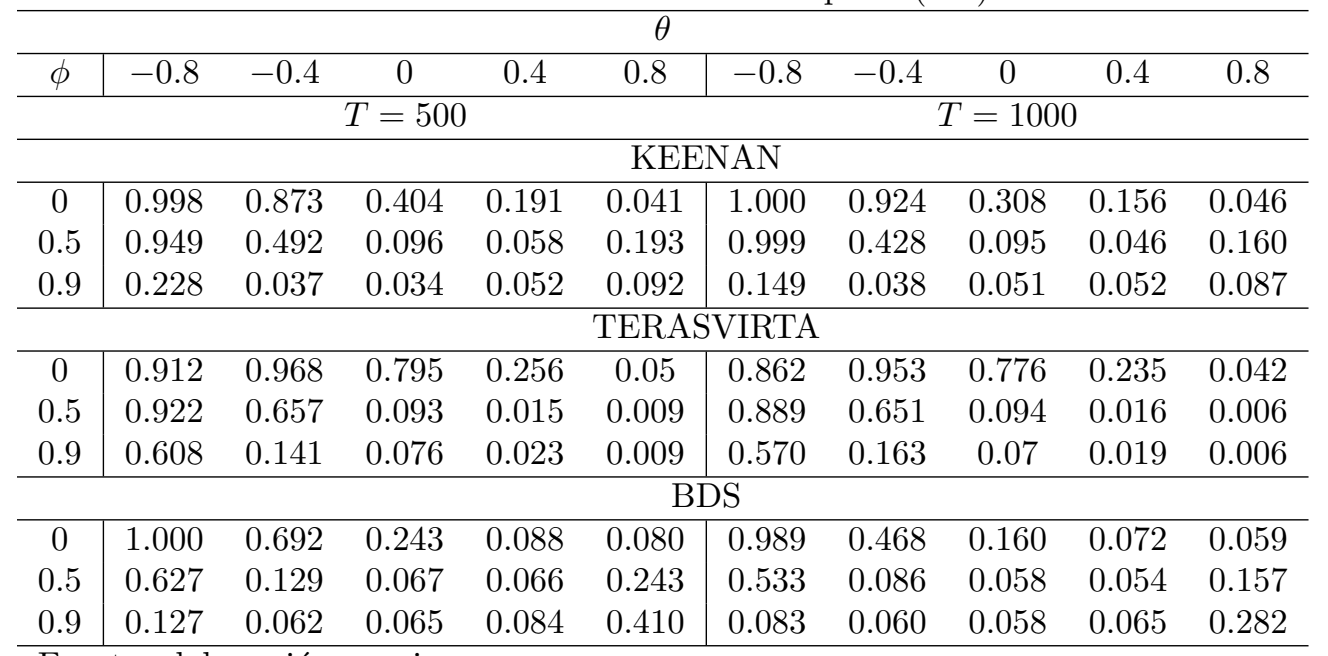

Fuente: elaboración propia. 
Tabla 5: Simulación con cambio de tendencia (LS)

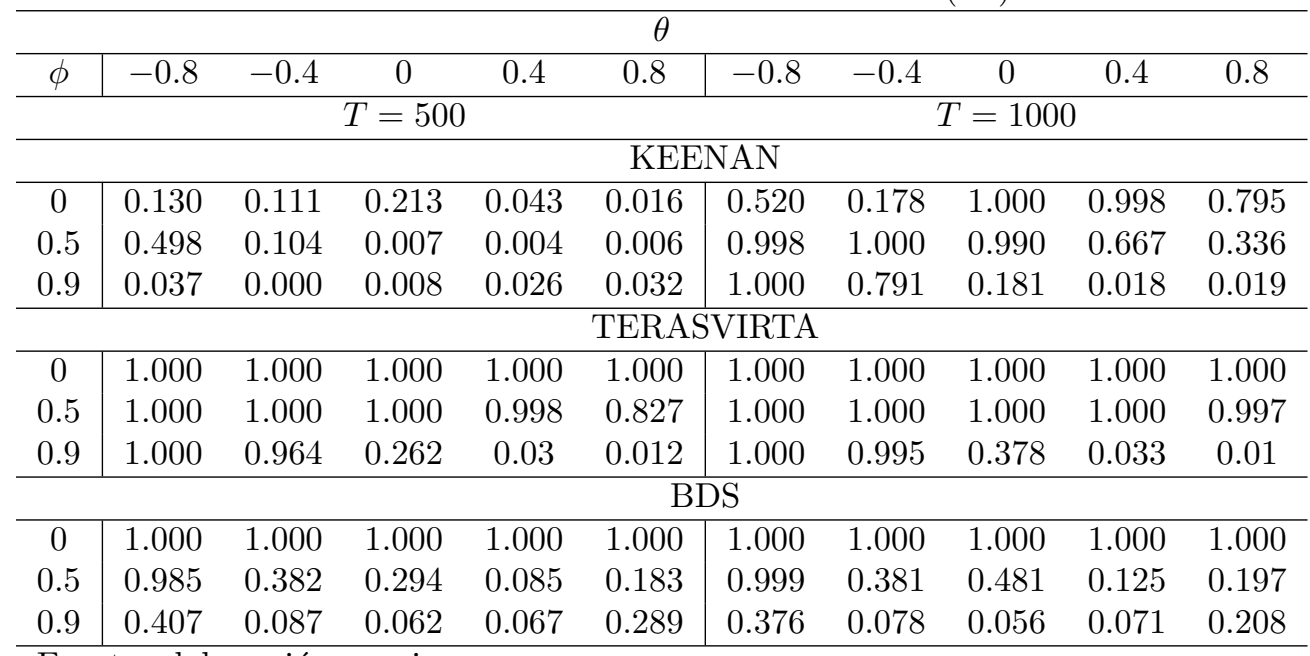

Fuente: elaboración propia.

\section{Detección de datos atípicos y pruebas de no li- nealidad para la serie de electricidad}

Los datos empleados corresponden a la serie diaria de los precios de la electricidad en Colombia. Se comienza desde el 2 de Enero de 2010 y se finaliza el 21 de Septiembre de 2018 para un total de 3185 observaciones 4 . En la Figura 2 se observa la evolución temporal de los precios. El análisis gráfico no muestra una tendencia creciente de los precios y no se observa una varianza incondicional inestable que cambie con el nivel de la serie. Por lo tanto no se realiza una transformación de tipo Box-Cox. Es evidente que para un periodo específico en el tiempo los precios y su volatilidad son inusualmente altos. Este periodo coincide con el fenómeno de El Niño que afecto al país a finales del 2015 y durante el 2016. Este hecho sugiere que el proceso se ve afectado por un evento exógeno.

Los contrates de no linealidad para la serie original se presentan en la Tabla 64A. Los tres contrastes rechazan la hipótesis nula de linealidad a un nivel de significancia del $5 \%$. El test BDS se realizó sobre los residuales del modelo $A R I M A(2,1,1)$.

Mediante la metodología propuesta por (Chen \& Liu 1993) se procede a detectar la presencia de datos atípicos y a estimar sus magnitudes. Para determinar la significancia de los coeficientes $\widehat{T}_{i}$ se usa como valor crítico 4.0, se fija en cuatro el máximo número de iteraciones internas. Para elegir los órdenes del polinomio autorregresivo y de medias móviles se emplea el criterio de información de Bayes. Se encontraron 74 datos atípicos significativos, en la Figura 3 se muestra su localización. El modelo que mejor se ajusta a los datos es el $A R I M A(1,0,0)$. Se

\footnotetext{
${ }^{4}$ Los datos empleados fueron obtenidos del portal web de la empresa XM.
} 


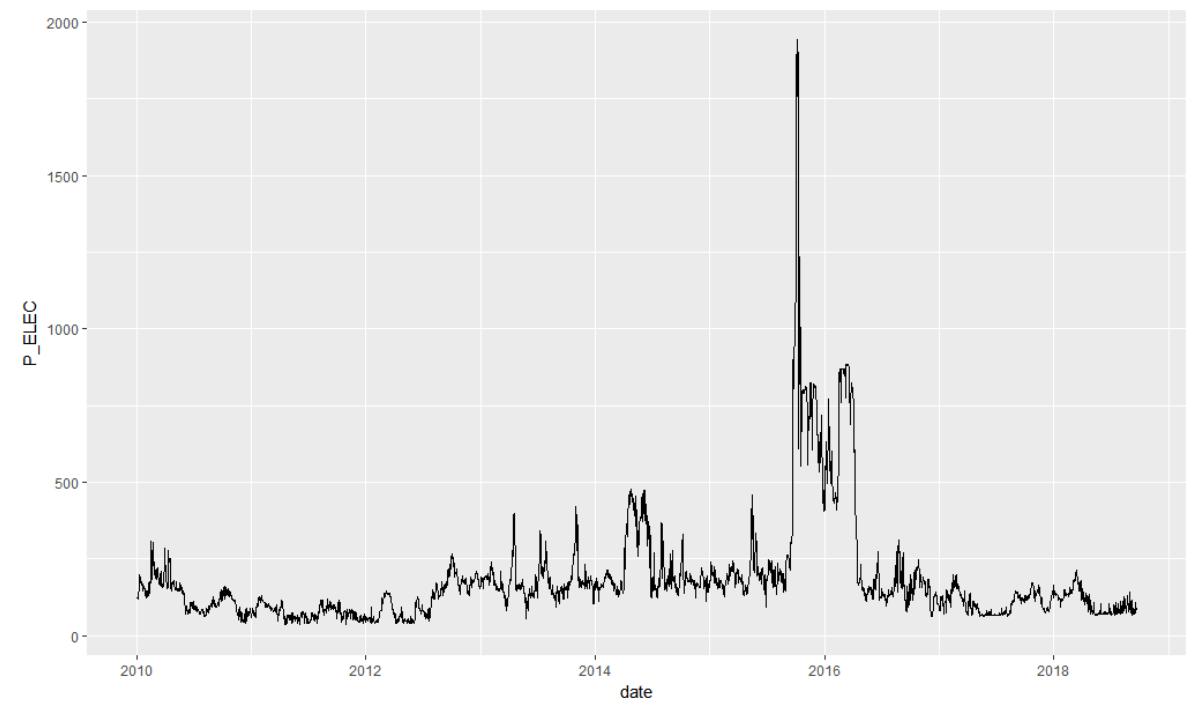

Figura 2: Precios de la electricidad en Colombia.

Tabla 6: Contrastes de no linealidad para la serie de electricidad

\begin{tabular}{lcc}
\hline \multicolumn{3}{l}{ A. Serie Original } \\
\hline Keenan & 46.724 & $(0.000)$ \\
Terasvirta & 75.574 & $(0.000)$ \\
BDS & 24.017 & $(0.000)$ \\
\hline B. Serie Corregida & \\
\hline Keenan & 0.024 & $(0.877)$ \\
Terasvirta & 10.652 & $(0.005)$ \\
BDS & 21.365 & $(0.000)$ \\
\hline \multicolumn{4}{l}{ Fuente: elaboración } &
\end{tabular}

Fuente: elaboración propia. 


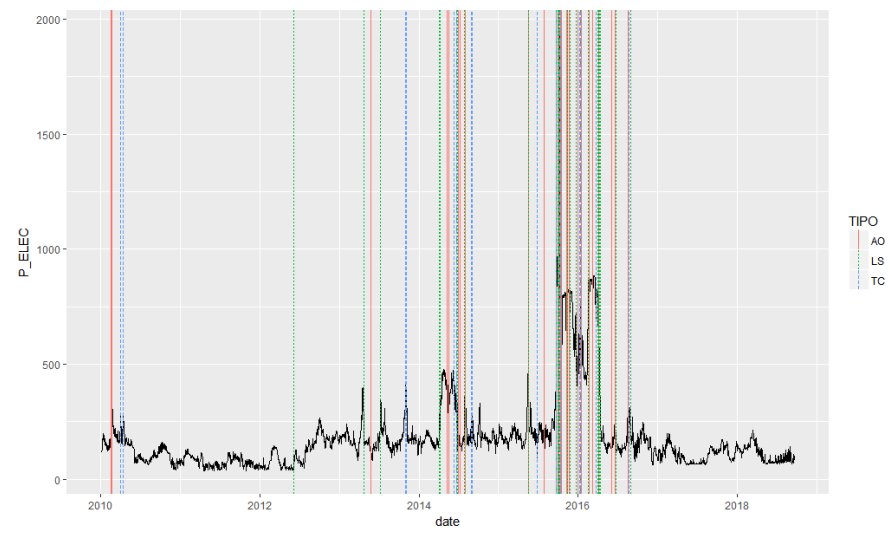

Figura 3: Localización de datos atípicos

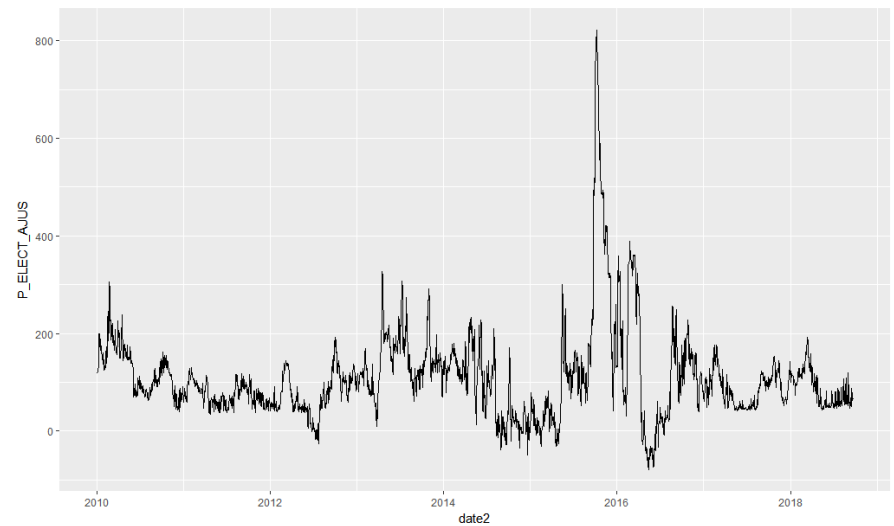

Figura 4: Serie corregida Precios de la electricidad.

realizan de nuevo los contrastes de no linealidad para la serie corregida eliminando el efecto de los datos atípicos, Figura 4 los resultados se presentan en la Tabla 6B. Como puede observarse, el contraste de Terasvirta y el test BDS sugieren que el precio de la electricidad en Colombia presenta una estructura no lineal en el proceso generador de datos. Por lo tanto, el uso de modelos que incorporen esta característica puede resultar positivo para obtener un mejor pronóstico de la serie.

\section{Conclusiones}

Es común que las series de tiempo económicas presenten cambios en su estructura dinámica debido a la existencia de eventos exógenos. Lo anterior representa un desafío para la correcta identificación del modelo. Esto es especialmente impor- 
tante cuando se determina si el proceso generador de datos es lineal o no lineal. En este trabajo se mostró que cuando se incorporan datos atípicos a una serie proveniente de un proceso lineal, el deterioro de las pruebas para aceptar hipótesis nula de linealidad es ampliamente significativo. Se señala entonces la importancia de incorporar en la modelación una metodología adecuada de tratamiento de atípicos. Para el caso del mercado eléctrico colombiano, se encuentra que la serie de precios está afectada por múltiples eventos exógenos de diferente naturaleza. Sin embargo, realizando un análisis de intervención y eliminando el efecto de atípicos, existe evidencia de no linealidad de carácter endógeno.

Recibido: $18 / 01 / 2020$

Aceptado: 26/05/2020 


\section{Referencias}

Barrientos, J., Rodas, E., Velilla, E., Lopera, M. \& Villada, F. (2012), 'Modelo para el pronóstico del precio de la energía eléctrica en colombia', (77).

Box, G. \& Jenkins, G. (1970), Time series analysis, forecasting and control, San Francisco, CA:

Brock, W. \& de Lima, P. (1995), 'Nonlinear time series, complexity theory, and finance.'

Brock, W., Dechert, D., Scheinkman, J. \& Lebaron, B. (1996), 'A test for independence based on the correlation dimension', Vol 15(3), 197-235.

Campbell, J., Lo, A. \& MacKinlay, C. (1997), The Econometrics of Financial Markets.

Castaño, E. \& Sierra, J. (2012), 'Sobre la existencia de una raíz unitaria en la serie de tiempo mensual del precio de la electricidad en colombia', (76).

Chen, C. \& Liu, L. (1993), 'Joint estimation of model parameters and outlier effects in time series', (88), 284-297.

Cochrane, J. H. (1991), '[pitfalls and opportunities: What macroeconomists should know about unit roots]: Comment', 6, 201-210.

Gassberger, P. \& Procaccia, I. (1983), 'Measuring the strangeness of strange attractors', Vol. 9, 189-208.

Hinich, M. J. (1982), 'Testing for gaussianity and linearity of a stationary time series', (3), 169-176.

Hsieh, D. (1995), 'Nonlinear dynamics in financial markets: evidence and implications', 51.

Keenan, D. (1985), 'A tukey nonadditivity-type test for time series', (72), 39-44.

Kyrtsou, C. \& Serletis, A. (2006), 'Univariate tests for nonlinear structure', (28), 154-168.

Lira, F., Muñoz, C., Nuñez, F. \& Cipriano, A. (2009), 'Short-term forecasting of electricity prices in the colombian electricity market', 3, 980-986.

López de Lacalle, J. (2017), Tsoutliers r package for automatic detection of outliers in time series, Technical report, Spain: CRAN.

Tabarez, E. (2017), Red neuronal versus modelo econométrico: una aplicación al pronóstico a la serie de precios de la energía eléctrica en colombia, Master's thesis.

Teräsvirta, T. (1996), 'Power properties of linearity tests for time series.', pp. 3-10. 
Teräsvirta, T., Tjøstheim, D. \& Granger, C. W. J. (1994), Aspects of modelling nonlinear time series.

Tsay, R. (2001), Analysis of Financial Time Series, New York: Wiley.

Tsay, R. S. (1986), 'Time series model specification in the presence of outliers', (81), 132-141.

van Dijk, D., Franses, P. H, . \& Lucas, A. (1999a), 'Testing for arch in the presence of additive outliers', 14, 539-562.

van Dijk, D., Franses, P. H, . \& Lucas, A. (1999b), 'Testing for smooth transition nonlinearity in the presence of outliers', 17(2), 217-235.

Velásquez, J. D. (2008), Construcción de Escenarios de Pronóstico del Precio de Eléctricidad en Mercados de Corto Plazo, PhD thesis.

Velásquez, J. D. \& Franco, C. J. (2010), 'Predicción de los precios de contratos de electricidad usando una red neuronal con arquitectura dinámica', 20(36), 714.

\section{A. Código: simulación modelo ARMA con datos atípicos}

Se simulan distintas series provenientes del modelo $A R M A(1,1)$ para distintos valores del parámetro autorregresivo y de medias móviles. Se añade el efecto del dato atípico mediante el comando outliers () de la librería outliers. Se consideran distintos tipos de atípicos: atípico innovacional (IO), atípico aditivo (AO), cambio temporal (TC) y cambio de tendencia (LS). A continuación se muestra el código usado para simular el modelo $\operatorname{ARMA}(\phi=0.5, \theta=-0.8)$ con un dato atípico innovacional. Se usan las pruebas de (Teräsvirta 1996), (Keenan 1985) y test BDS para contrastar la hipótesis nula de linealidad.

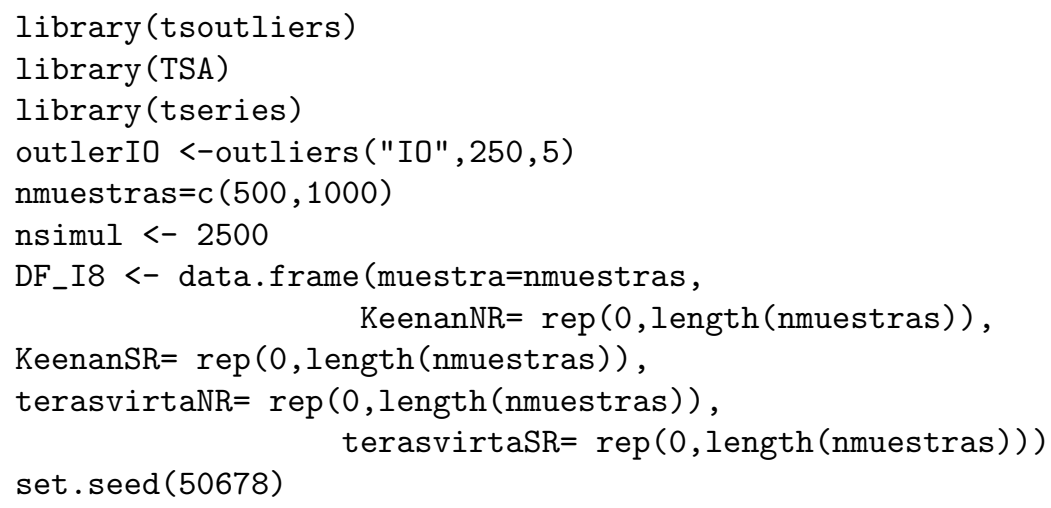




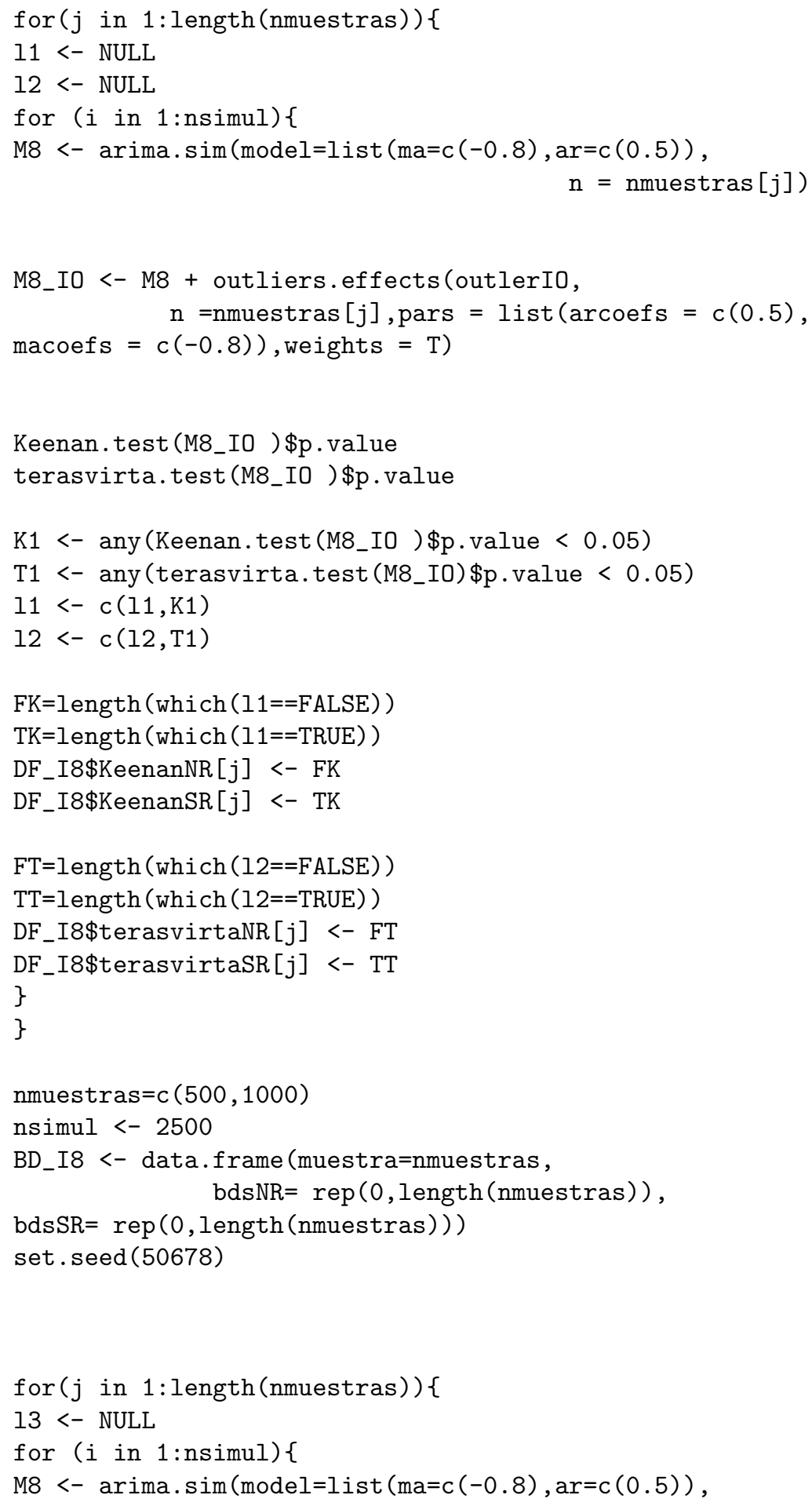




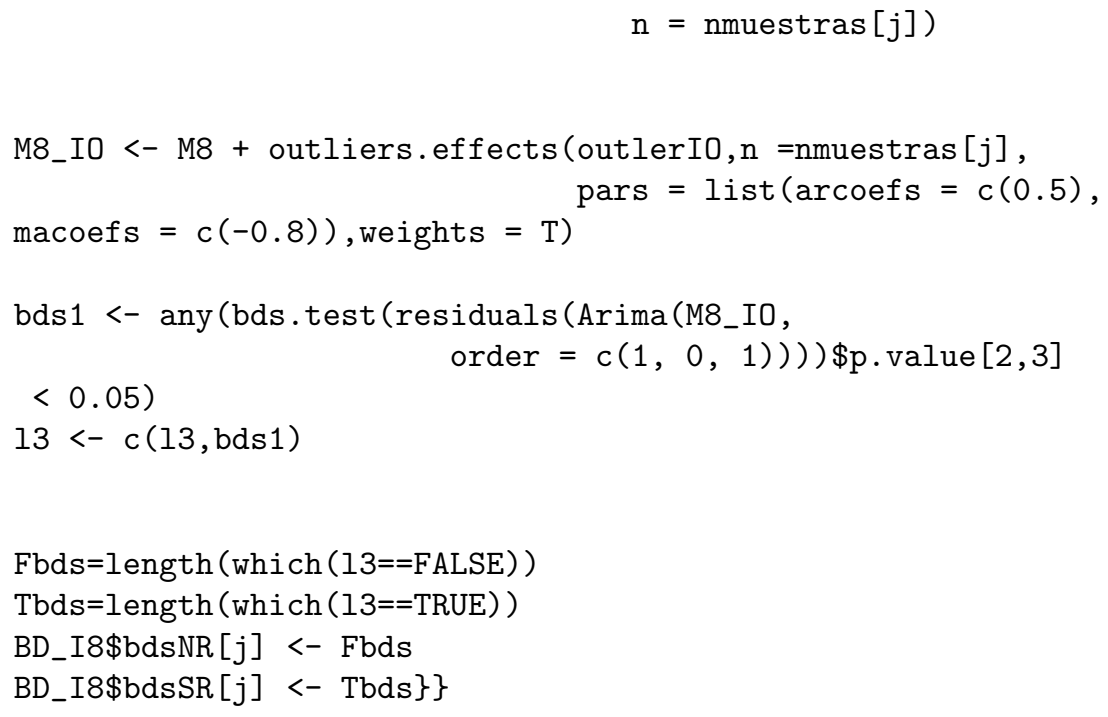

